\title{
Current and Future Perspectives on Light Therapy Using Wearable Devices
}

\author{
Jiye Lee, Jooyoung Lee, and Seog Ju Kim \\ Department of Psychiatry, Sungkyunkwan University School of Medicine, Samsung Medical Center, Seoul, Korea
}

\begin{abstract}
Circadian rhythm is the internal clock that regulates physiological functions, including the sleep-wake cycle. Advancements in technology and lifestyle have negatively impacted the circadian rhythm, resulting in numerous sleep disorders. Because light is the most important "zeigeber" for synchronizing the circadian system, light therapy is effective for circadian rhythm sleep disorders. We compared light therapy glasses with a traditional light therapy box, summarized the characteristics of various light therapy glasses, and proposed future applications of wearable sleep technologies in light therapy.

Keywords: Light therapy; Wearable devices; Circadian rhythm

Received: August 21, 2021 Revised: September 5, 2021 Accepted: September 6, 2021

Corresponding author: Seog Ju Kim, MD, PhD, Department of Psychiatry, Sungkyunkwan University School of Medicine, Samsung Medical Center, 81 Irwon-ro, Gangnam-gu, Seoul 06351, Korea.

Tel: 82-2-3410-3589, Fax: 82-2-3410-0050, E-mail: ksj7126@skku.edu

@ This is an Open Access article distributed under the terms of the Creative Commons Attribution Non-Commercial License (https://creativecommons.org/licenses/bync/4.0) which permits unrestricted non-commercial use, distribution, and reproduction in any medium, provided the original work is properly cited.
\end{abstract}

\section{SLEEP AND CIRCADIAN RHYTHM}

Circadian rhythm is the biological clock that regulates several physiological functions of the body, including the sleep-wake cycle, immunity, hormone secretion, and temperature. The circadian rhythm consists of a self-sustaining cycle, lasting for approximately 24 hours, generated by a "master clock" in the hypothalamic suprachiasmatic nucleus (SCN). This cycle is entrained daily by zeitgeber (time giver) signals, such as light, diet, and exercise [1-3]. Light exposure plays the most important role in synchronizing our internal clock. In humans, light is perceived not only by rods and cones but also by a subset of intrinsically photosensitive retinal ganglion cells (ipRGCs). Axons of the ipRGCs synapse at the hypothalamus and other subcortical regions [4,5]. Non-visual input is transmitted by the ipRGCs to the circadian clock in the SCN. The ipRGCs express the photopigment melanopsin, which is sensitive to short-wavelength light. Therefore, ipRGCs synchronize the circadian clock to the 24-hour light-dark cycle, and regulate sleep, alertness, pupillary size, and various other physiological functions [6,7].

Light is transmitted from the retina to the SCN through the aforementioned pathway, which also inhibits the production of melatonin in the pineal gland [8]. Melatonin is mainly produced at night, and promotes sleep by switching the biological day (state of arousal and increased energy intake and consumption) to the biological night (e.g., sleep, low temperature, and energy conservation) [9]. Simultaneously, cortisol production increases, reaching a maximum at 30 minutes after awakening, which is called the cortisol awakening response. Both sleep-wake and light-dark cycles, as well as the quantity and quality of sleep, affect cortisol secretion [10].

\section{EFFECTS OF MODERN LIFE ON THE CIRCADIAN RHYTHM}

Advancements in technology have allowed people to work at any time of the day, travel across time zones, and use electronic devices, such as television, computer, and cellular phones. Although such changes have made life more comfortable, they have harmful effects on the sleep pattern, duration, and quality [11]. Approximately 20\% of the population work outside of traditional working hours [12] and have various schedules, such as early working hours, 12-hour work shifts, and night work. Such schedules lead to excessive sleepiness and sleep disruption [13]. In addition, flexible work schedules are associated with reduced sleep quality [14]. Jetlag is an important problem for aircrew and travelers [15]. 
Adolescents spend a large amount of time during the day and at bedtime using electronic devices; excessive use of electronic media not only reduces sleep duration and quality but also negatively affects daily functioning and mood [16,17].

Additionally, lifestyle factors are closely related to sleep hygiene. Reduced physical activity and a sedentary lifestyle (e.g., sitting or reclining with low energy expenditure) increase the risks of insomnia and sleep disturbance. Dietary changes (e.g., high-fat diets), overweight, and obesity are also related to sleep deprivation and disturbance [11]. Caffeine is widely used to relieve daytime sleepiness; however, it leads to poor sleep habits and quality [18].

\section{LIGHT THERAPY IN SLEEP DISORDERS}

Because light is a critical factor in regulating sleep and wakefulness, it has been used for the treatment of sleep disorders. Light therapy is effective for sleep disorders, particularly circadian rhythm sleep disorders (CRSDs) and insomnia [19]. The effects of light therapy depend on the duration and time of exposure, intensity, and wavelength of light $[20,21]$.

CRSDs are characterized by dysfunction of the circadian rhythm (e.g., delayed and advanced sleep phase disorders) or desynchronization of the circadian rhythm and external environment (e.g., shift work and jet lag sleep-wake disorders) [22]. In delayed sleep phase disorder (DSPD), sleep onset occurs much later than the desired sleep time. DSPD patients experience sleep-onset insomnia and excessive sleepiness in the morning [23], due to internal (e.g., longer circadian cycle or impaired ability to compensate for phase advances) and external (e.g., schoolwork) factors that lead to progressively increasing delays in sleep onset [24]. Such patients benefit from light therapy in the morning, shortly after awakening, to advance the sleep onset. They are recommended to avoid exposure to bright artificial light in the evening, before bedtime. Early-morning light therapy, coupled with light restriction in the evening, successfully advances the circadian rhythm in DSPD patients $[25,26]$. In contrast, advanced sleep phase disorder (ASPD) is characterized by sleep onset that occurs earlier than the desired bedtime [27]. ASPD patients experience excessive sleepiness in the evening and complain of early morning awakening, due to a short endogenous circadian period or reduced capability for phase delay shifts [23]. ASPD is treated with timed light exposure in the evening, just prior to bedtime, which delays the sleep-wake cycle. Evening light therapy delays the sleep-wake cycle and improves early-morning awakening insomnia [28-30]. ASPD patients are recommended to avoid exposure to bright light in the morning hours, after awakening, to prevent undesirable phase advance shifts [31]. ASPD predominantly affects the older population [32]; evening light therapy is a good alternative to medications, such as hypnotics, for the treatment of ASPD.

Jetlag is a type of CRSD that occurs after transmeridian jet travel and is characterized by insomnia, excessive daytime sleepiness, malaise, and somatic symptoms. A day is required to resynchronize the circadian rhythm for each time zone crossed [33]. Criti- cally timed bright light exposure reduces the symptoms of jetlag. Bright light synchronizes the circadian rhythm and enhances the light signals to the brain, which are appropriate for the environment. Therefore, bright light synchronizes the body's circadian rhythm to the external surroundings [34]. The method of light exposure used for the treatment of jet lag depends on the direction of flight (westward or eastward) and number of time zones crossed. Jetlag after westward flights (e.g., London to New York) can be avoided by staying awake during day and sleeping at night. Conversely, to prevent jetlag during travel from New York to London, an individual would have to stay awake and avoid sunlight during the day, and be exposed to sunlight during the afternoon [35].

Shift work is associated with several problems, including accidents, cardiovascular and gastrointestinal diseases, cancer, and depression [36-38]. A significant proportion of shift workers develop shift work disorder, characterized by excessive sleepiness and insomnia [39]. The treatment of shift work disorder depends on the type of work, e.g., night shift workers may be treated with exposure to bright light during the night shift $(4,000-7,000$ lux for 4 hours) to enhance sleep and performance [40]. Intermittent light exposure ( 15 minutes $\times 4$ times) at night, along with avoidance of light using sunglasses during morning, improves alertness and performance during the night shift and allows sufficient daytime sleep after work [41]. Most studies of light therapy have mainly focused on night shift workers, even though many early morning and rotational shift workers also experience shift work disorders. Further studies are required to determine the optimal light therapy for these individuals [23].

\section{COMPARISON BETWEEN LIGHT THERAPY USING A TRADITIONAL BOX OR GLASSES}

Recently, light therapy glasses have been developed as substitutes for the traditional light box. Light therapy glasses are similar to regular eyeglasses, except that they cast specific intensity and wavelengths of light beam to eyes or faces. Some devices are band-type glasses emitting light downward from sources in the glasses frame. Others are similar to the regular eyeglasses, which have regular glasses frame or lens. Light may be emitted from lower part of frame or lens itself.

Only a few studies have compared a traditional light therapy box with light therapy glasses. Light therapy devices emit light of different wavelengths and intensity, which have varying effects on physiology and behavior. A traditional light therapy box emits a polychromatic white light; whereas light therapy glasses emit a blue-enriched white light or monochromatic blue light. Although both modalities demonstrate similar benefits, several studies have reported that blue-enriched light is more effective [42]. Compared to the standard white light, blue-enriched light improves subjective sleepiness, alertness, and performance $[43,44]$. Therefore, blue-enriched light glasses have stronger effects, as compared to the traditional light therapy box. Because users are seated at a 
distance, a light box emits light of at least 10,000 lux, which is very bright and intrusive. In comparison, light therapy glasses emit light of 500-2,000 lux, which allows users to simultaneously continue their daily activities without irritation [45].

The light therapy boxes are large and bulky, which makes outdoor use inconvenient. The users are required to remain stationary and view the box for a few minutes every morning, which interferes with their daily routine. Light therapy glasses are portable and allow freedom of movement during the intervention. While wearing the glasses, patients are able to continue their daily activities (e.g., eat breakfast, brush their teeth, read a book, watch television, exercise, drive, and work on the computer) [46].

The light therapy box can be tilted to adjust the angle [47], which is unnecessary for the wearable light therapy glasses. The light therapy glasses have a longer battery life compared to the light therapy box. Furthermore, the box only provides a timer function; whereas the glasses can be synchronized with mobile applications to collect personalized data. In summary, light therapy glasses, despite being more expensive, have many advantages over the traditional light therapy box.

\section{FEATURES OF LIGHT THERAPY GLASSES}

Light therapy glasses have been used to treat seasonal affective disorder, CRSDs, and jetlag. Users wear the glasses for around 30 min in the morning or after lunch, which provides benefits similar to those of a short nap [48]. All glasses filter ultraviolet and infrared light to avoid ocular damage. There are many unique features of commercially available light therapy glasses. Using information provided on the manufacturer's website, we compared the features of different light therapy glasses (Table 1) [49-55].

Light therapy glasses emit different light spectrums. Luminette 3 (Lucimed SA) and Pocket Sky (Active Wearables GmbH) emit blue-enriched white light that peaks at $468-470 \mathrm{~nm}$. Propeaq (Chrono Eyewear BV) emits a 468-nm monochromatic blue light. Ayo (Novalogy Inc.) and Sula (Sula Health Ltd.) emit blueturquoise light, at approximately $470-480 \mathrm{~nm}$. Re-timer (Re-Timer Pty Ltd.) and Pegasi II (Shenzhen Qianhai Icecold IT Co., Ltd.) emit light with a greater green component than blue (bluegreen light, $500 \mathrm{~nm}$ ). The optimal source for light therapy is unclear. The effectiveness of blue monochromatic light has been es- tablished. Because retinal ganglion cells that contain the blue light-sensitive photopigment project to the $\mathrm{SCN}$, exposure to monochromatic blue light $(460 \mathrm{~nm})$ is more effective than green light $(555 \mathrm{~nm})$ at phase-resetting the circadian system [56]. However, further studies are required to determine the efficacy of different wavelengths.

Some light therapy glasses connect to a mobile application to provide a user-friendly experience, including Luminette 3 (MyLuminette), Pegasi II (PEGASI), Propeaq (Propeaq, Propeaq shift work), Ayo (goAYO), and Sula (SULA). The goAYO application provides several programs (energy, sleep, and travel programs) that can be used depending on the specific requirements. For example, the energy program allows users to revitalize after a night of insufficient sleep or after lunch. The sleep program helps users to phase-shift the circadian rhythm. The travel program was designed to provide the optimal duration of light therapy to treat jetlag [57].

Some wearables, such as Pegasi II, Propeaq, and Sula, filter harmful blue light. Users are recommended to wear these glasses before bedtime. Treatment with morning bright light and shortwavelength filter glasses in the evening improves sleepiness, morning mood, and night awakenings [58]. Light avoidance during evening is associated with increased total sleep time [59].

The beam of most glasses, including Luminette 3, Pegasi II, Ayo, Propeaq, and Pocket Sky, is angled downward, simulating the angle formed by sunlight and allowing the users to focus their vision at their will. The light-emitting diode (LED) of Re-timer and Sula is placed below the level of the eyes to avoid blockage by the eyebrows.

The light therapy glasses have different weights. Re-timer (75 g) and Luminette 3 (50 g) weigh more than other glasses. Propeaq (41 g), Pegasi II (36 g), and Ayo (31 g) have weights similar to those of regular sunglasses (30-40 g), and Pocket Sky (12 g) has the lowest weight. Light-weight glasses are easier to wear during work.

Glasses without charging cases provide battery life of about a week (Propeaq: 5 days; Pegasi II: 7 days; Sula: 7 days; Re-timer: 8 days; and Luminette 3: 10 days). Glasses with charging cases provide a longer battery life. Ayo can be charged wirelessly, and provides a battery life of 26 days. Pocket Sky provides a battery life of 14 days, with its charging case. Because light therapy glasses are designed to be portable, a long battery life is necessary for them.

Table 1. Features of light therapy glasses

\begin{tabular}{|c|c|c|c|c|c|}
\hline Device & Light spectrum & Session duration (min) & Mobile application & Weight (g) & Battery life (d) \\
\hline Luminette 3 [49] & White (blue-enriched) & $20-45$ & O (MyLuminette) & 50 & 10 \\
\hline Pegasi II [50] & Blue-green & 30 & O (PEGASI) & 36 & 7 \\
\hline Re-timer [51] & Blue-green & $30-60$ & $\mathrm{X}$ & 75 & 8 \\
\hline Propeaq [53] & Monochromatic blue & 30 & $\begin{array}{c}\text { O (Propeaq, } \\
\text { Propeaq Shiftwork) }\end{array}$ & 41 & 5 \\
\hline Ayo [52] & Blue-turquoise & 20 & $\mathrm{O}$ (goAYO) & 31 & 26 \\
\hline Pocket Sky [54] & White (blue-enriched) & 20 & $\mathrm{X}$ & 12 & 14 \\
\hline Sula [55] & Blue-turquoise & 30 & O (SULA) & N/A & 7 \\
\hline
\end{tabular}


Many light therapy glasses with different features are commercially available. These glasses vary in terms of wavelength of light beams, associated mobile applications, design, weight, battery life, and cost-effectiveness.

\section{FUTURE WEARABLE SLEEP TECHNOLOGIES FOR LIGHT THERAPY}

There is growing interest in the use of wearable technologies for sleep disorders [60]. Among the current devices, wrist-worn devices are most commonly used, and include first-generation wearables, which use an accelerometer-type sensor to collect motionbased data (e.g., Jawbone UP, Fitbit Ultra, and Fitbit Flex), and new-generation wearables. New-generation wearables, including smart watches (e.g., E4 wristband, Fitbit Sense, Vivoactive 4, and Galaxy Watch 3), record bio-signals (such as electrocardiography, heart rate and variability thereof, respiratory rate, oxygen saturation, skin temperature, and skin conductance), as well as provide information about sleep duration, stage, and quality. More importantly, wrist wearables can provide information regarding circadian rhythm of individuals, by assessing the time and duration of individual's diurnal activity, rest or light exposure. Additionally, ambulatory electrophysiology requires the use of wearable devices to measure sleep on electroencephalography. There are few data on the validity and clinical use of wearable devices. Given the improvements in the validity, accuracy, and utility of these devices, wearable sleep technologies will contribute to our understanding of sleep patterns and quality. Technological developments in wearable devices have improved light therapy for CRSD patients. Based on personal data obtained from the wearable devices, light therapy is customized for the patients. Installation of light sensors on the wrist-worn devices would extend their application to phase-shifts in the circadian rhythm [61,62]. Furthermore, virtual and augmented reality devices would allow better evaluation of sleep disorders and response to light therapy. Ultimately, wearable sleep technologies are expected to improve the treatment of sleep disorders significantly.

\section{Acknowledgments}

This work was supported by the Technology Innovation Infrastructure program through the Korea Institute for Advancement of Technology by The Ministry of Trade, Industry and Energy [No. P0014279], and a grant of the Korea Health Technology R\&D Project through the Korea Health Industry Development Institute (KHIDI), funded by the Ministry of Health \& Welfare, Republic of Korea (No. HR21C0885).

\section{Conflicts of Interest}

The authors have no potential conflicts of interest to disclose.

\section{Author Contributions}

Conceptualization: Seog Ju Kim. Data curation: Jiye Lee. Formal analysis: Jiye Lee. Funding acquisition: Seog Ju Kim. Investiga- tion: Jiye Lee, Jooyoung Lee. Methodology: Jiye Lee, Jooyoung Lee. Project administration: Seog Ju Kim. Resources: Seog Ju Kim. Software: Jiye Lee. Supervision: Seog Ju Kim. Validation: Jooyoung Lee. Visualization: Jiye Lee. Writing_original draft: Jiye Lee. Writing—review \& editing: Jooyoung Lee, Seog Ju Kim.

\section{ORCID iDs}

Jiye Lee (D)

https://orcid.org/0000-0002-7733-2404

Jooyoung Lee (D)

https://orcid.org/0000-0002-8774-7128

Seog Ju Kim (B)

https://orcid.org/0000-0003-2467-5451

\section{REFERENCES}

1. Golombek DA, Rosenstein RE. Physiology of circadian entrainment. Physiol Rev 2010;90:1063-1102.

2. Youngstedt SD, Elliott JA, Kripke DF. Human circadian phase-response curves for exercise. J Physiol 2019;597:2253-2268.

3. Pickel L, Sung HK. Feeding rhythms and the circadian regulation of metabolism. Front Nutr 2020;7:39.

4. Brown TM. Melanopic illuminance defines the magnitude of human circadian light responses under a wide range of conditions. J Pineal Res 2020;69: e12655.

5. Do MTH. Melanopsin and the intrinsically photosensitive retinal ganglion cells: biophysics to behavior. Neuron 2019;104:205-226.

6. Wirz-Justice A, Skene DJ, Münch M. The relevance of daylight for humans. Biochem Pharmacol 2021;191:114304.

7. Spitschan M. Melanopsin contributions to non-visual and visual function. Curr Opin Behav Sci 2019;30:67-72.

8. Vriend J, Reiter RJ. Melatonin feedback on clock genes: a theory involving the proteasome. J Pineal Res 2015;58:1-11.

9. Cipolla-Neto J, Amaral FGD. Melatonin as a hormone: new physiological and clinical insights. Endocr Rev 2018;39:990-1028.

10. Niu SF, Chung MH, Chu H, Tsai JC, Lin CC, Liao YM, et al. Differences in cortisol profiles and circadian adjustment time between nurses working night shifts and regular day shifts: a prospective longitudinal study. Int J Nurs Stud 2015;52:1193-1201.

11. Shochat T. Impact of lifestyle and technology developments on sleep. Nat Sci Sleep 2012;4:19-31.

12. Potter GDM, Wood TR. The future of shift work: circadian biology meets personalised medicine and behavioural science. Front Nutr 2020;7:116.

13. Boivin DB, Boudreau P. Impacts of shift work on sleep and circadian rhythms. Pathol Biol (Paris) 2014;62:292-301.

14. Martens MFJ, Nijhuis FJN, van Boxtel MPJ, Knottnerus JA. Flexible work schedules and mental and physical health. A study of a working population with non-traditional working hours. J Organ Behav 1999;20:35-46.

15. Cho K, Ennaceur A, Cole JC, Suh CK. Chronic jet lag produces cognitive deficits. J Neurosci 2000;20:RC66.

16. Hysing M, Pallesen S, Stormark KM, Jakobsen R, Lundervold AJ, Sivertsen B. Sleep and use of electronic devices in adolescence: results from a large population-based study. BMJ Open 2015;5:e006748.

17. Royant-Parola S, Londe V, Tréhout S, Hartley S. The use of social media modifies teenagers' sleep-related behavior. L'Encéphale 2018;44:321-328.

18. Snel J, Lorist MM. Effects of caffeine on sleep and cognition. Prog Brain Res 2011;190:105-117.

19. van Maanen A, Meijer AM, van der Heijden KB, Oort FJ. The effects of light therapy on sleep problems: a systematic review and meta-analysis. Sleep Med Rev 2016;29:52-62.

20. Rahman SA, Wright KP Jr, Lockley SW, Czeisler CA, Gronfier C. Characterizing the temporal dynamics of melatonin and cortisol changes in response to nocturnal light exposure. Sci Rep 2019;9:19720.

21. Leger D, Duforez F, Gronfier C; et le Groupe consensus chronobiologie et sommeil de la Société française de recherche et médecine du sommeil 
(SFRMS). Treating circadian sleep-wake disorders by light. Presse Med 2018;47(11-12 Pt 1):1003-1009.

22. Zee PC, Attarian H, Videnovic A. Circadian rhythm abnormalities. Continuum (Minneap Minn) 2013;19:132-147.

23. Dodson ER, Zee PC. Therapeutics for circadian rhythm sleep disorders. Sleep Med Clin 2010;5:701-715.

24. Wyatt JK. Delayed sleep phase syndrome: pathophysiology and treatment options. Sleep 2004;27:1195-1203.

25. Rosenthal NE, Joseph-Vanderpool JR, Levendosky AA, Johnston SH, Allen $\mathrm{R}$, Kelly KA, et al. Phase-shifting effects of bright morning light as treatment for delayed sleep phase syndrome. Sleep 1990;13:354-361.

26. Figueiro MG. Delayed sleep phase disorder: clinical perspective with a focus on light therapy. Nat Sci Sleep 2016;8:91-106.

27. Ondzé B, Espa F, Ming LC, Chakkar B, Besset A, Billiard M. Advanced sleep phase syndrome. Rev Neurol (Paris) 2001;157(11 Pt 2):S130-S134.

28. Palmer CR, Kripke DF, Savage HC Jr, Cindrich LA, Loving RT, Elliott JA. Efficacy of enhanced evening light for advanced sleep phase syndrome. Behav Sleep Med 2003;1:213-226.

29. Lack L, Wright H, Kemp K, Gibbon S. The treatment of early-morning awakening insomnia with 2 evenings of bright light. Sleep 2005;28:616-623.

30. Lack L, Wright $H$. The effect of evening bright light in delaying the circadian rhythms and lengthening the sleep of early morning awakening insomniacs. Sleep 1993;16:436-443.

31. Auger RR, Burgess HJ, Emens JS, Deriy LV, Thomas SM, Sharkey KM. Clinical practice guideline for the treatment of intrinsic circadian rhythm sleepwake disorders: advanced sleep-wake phase disorder (ASWPD), delayed sleep-wake phase disorder (DSWPD), non-24-hour sleep-wake rhythm disorder (N24SWD), and irregular sleep-wake rhythm disorder (ISWRD). An update for 2015: an American Academy of Sleep Medicine clinical practice guideline. J Clin Sleep Med. 2015;11:1199-1236.

32. Suzuki K, Miyamoto M, Hirata K. Sleep disorders in the elderly: diagnosis and management. J Gen Fam Med 2017;18:61-71.

33. Sack RL. The pathophysiology of jet lag. Travel Med Infect Dis 2009;7:102110.

34. Parry BL. Jet lag: minimizing it's effects with critically timed bright light and melatonin administration. J Mol Microbiol Biotechnol 2002;4:463-466.

35. Herxheimer A, Waterhouse J. The prevention and treatment of jet lag. BMJ 2003;326:296-297.

36. Kecklund G, Axelsson J. Health consequences of shift work and insufficient sleep. BMJ 2016;355:15210.

37. Knutsson A. Health disorders of shift workers. Occup Med (Lond) 2003;53: 103-108.

38. Drake CL, Roehrs T, Richardson G, Walsh JK, Roth T. Shift work sleep disorder: prevalence and consequences beyond that of symptomatic day workers. Sleep 2004;27:1453-1462.

39. Wickwire EM, Geiger-Brown J, Scharf SM, Drake CL. Shift work and shift work sleep disorder: clinical and organizational perspectives. Chest 2017; 151:1156-1172.

40. Dawson D, Encel N, Lushington K. Improving adaptation to simulated night shift: timed exposure to bright light versus daytime melatonin administration. Sleep 1995;18:11-21.

41. Smith MR, Fogg LF, Eastman CI. Practical interventions to promote circadian adaptation to permanent night shift work: study 4. J Biol Rhythms 2009; 24:161-172.

42. Comtet H, Geoffroy PA, Kobayashi Frisk M, Hubbard J, Robin-Choteau L, Calvel L, et al. Light therapy with boxes or glasses to counteract effects of acute sleep deprivation. Sci Rep 2019;9:18073.

43. Sletten TL, Ftouni S, Nicholas CL, Magee M, Grunstein RR, Ferguson S, et al. Randomised controlled trial of the efficacy of a blue-enriched light intervention to improve alertness and performance in night shift workers. Occup Environ Med 2017;74:792-801.

44. Sletten TL, Raman B, Magee M, Ferguson SA, Kennaway DJ, Grunstein RR, et al. A blue-enriched, increased intensity light intervention to improve alertness and performance in rotating night shift workers in an operational setting. Nat Sci Sleep 2021;13:647-657.

45. Mann J. Best light therapy glasses and wearables for sleep [Internet]. Available at: https://sleepgadgets.io/best-light-therapy-glasses-wearables/. Accessed July 28, 2021.

46. Kirschbaum-Lesch I, Gest S, Legenbauer T, Holtmann M. Feasibility and efficacy of bright light therapy in depressed adolescent inpatients. Z Kinder Jugendpsychiatr Psychother 2018;46:423-429.

47. Klein S, Wood B. 10 Top-rated light therapy boxes to use while you're stuck inside all day [Internet]. Available at: https://www.health.com/depression/ best-light-therapy-boxes. Accessed July 28, 2021.

48. Slama H, Deliens G, Schmitz R, Peigneux P, Leproult R. Afternoon nap and bright light exposure improve cognitive flexibility post lunch. PLoS One 2015;10:e0125359.

49. Lucimed SA. Luminette [Internet]. Available at: https://www.myluminette. com/en-ca/luminette. Accessed July 28, 2021.

50. Shenzhen Qianhai Icecold IT. PEGASI [Internet]. Available at: http://www. pegasiglasses.com/Product/. Accessed July 28, 2021.

51. Re-Timer Pty Ltd. Re-Timer [Internet]. Available at: https://www.re-timer. com. Accessed July 28, 2021.

52. Novalogy Inc. AYO [Internet]. Available at: https://goayo.com/ayo-glasses. Accessed July 28, 2021.

53. Chrono Eyewear BV. PROPEAQ [Internet]. Available at: https://www.propeaq.com/en/light-glasses-2/. Accessed July 28, 2021.

54. Active Wearables GmbH. Pocket sky [Internet]. Available at: https://www. pocket-sky.com. Accessed July 28, 2021.

55. Sula Health Ltd. Sula [Internet]. Available at: https://www.sulasleep.com/ sula-glasses. Accessed July 28, 2021.

56. Gooley JJ. Treatment of circadian rhythm sleep disorders with light. Ann Acad Med Singap 2008;37:669-676

57. Mann J. Super review: Ayo vs Pocket Sky vs Luminette V3 light therapy wearables [Internet]. Available at: https://sleepgadgets.io/ayo-pocket-skyluminette-3-super-review. Accessed July 28, 2021.

58. Formentin C, Carraro S, Turco M, Zarantonello L, Angeli P, Montagnese S. Effect of morning light glasses and night short-wavelength filter glasses on sleep-wake rhythmicity in medical inpatients. Front Physiol 2020;11:5

59. Faulkner SM, Bee PE, Meyer N, Dijk DJ, Drake RJ. Light therapies to improve sleep in intrinsic circadian rhythm sleep disorders and neuro-psychiatric illness: a systematic review and meta-analysis. Sleep Med Rev 2019;46:108-123.

60. de Zambotti M, Cellini N, Menghini L, Sarlo M, Baker FC. Sensors capabilities, performance, and use of consumer sleep technology. Sleep Med Clin 2020;15:1-30.

61. Depner CM, Cheng PC, Devine JK, Khosla S, de Zambotti M, Robillard R, et al. Wearable technologies for developing sleep and circadian biomarkers: a summary of workshop discussions. Sleep 2020;43:zsz254.

62. de Zambotti M, Cellini N, Goldstone A, Colrain IM, Baker FC. Wearable sleep technology in clinical and research settings. Med Sci Sports Exerc 2019;51:1538-1557. 